\title{
Characterising the magnetic fields of the Herbig Ae/Be stars HD 97048, HD 150193, HD 176386, and MWC 480 *
}

\author{
S. Hubrig ${ }^{1}$, M. Schöller ${ }^{2}$, I. Ilyin ${ }^{1}$, C. R. Cowley ${ }^{3}$, Z. Mikulášek ${ }^{4,5}$, B. Stelzer ${ }^{6}$, M. A. Pogodin ${ }^{7,8}$, \\ R. V. Yudin ${ }^{7,8}$, and M. Curé ${ }^{9}$
}

\author{
${ }^{1}$ Leibniz-Institut für Astrophysik Potsdam (AIP), An der Sternwarte 16, 14482 Potsdam, Germany \\ e-mail: shubrig@aip.de \\ 2 European Southern Observatory, Karl-Schwarzschild-Str. 2, 85748 Garching bei München, Germany \\ 3 Department of Astronomy, University of Michigan, Ann Arbor, MI 48109-1042, USA \\ 4 Department of Theoretical Physics and Astrophysics, Masaryk University, Brno, Czech Republic \\ 5 Observatory and Planetarium of J. Palisa, VŠB - Technical University, Ostrava, Czech Republic \\ 6 INAF-Osservatorio Astronomico di Palermo, Piazza del Parlamento 1, 90134 Palermo, Italy \\ 7 Pulkovo Observatory, 196140 Saint-Petersburg, Russia \\ ${ }^{8}$ Isaac Newton Institute of Chile, Saint-Petersburg Branch, Russia \\ 9 Departamento de Física y Astronomía, Facultad de Ciencias, Universidad de Valparaíso, Chile
}

Received 4 June 2011 / Accepted 16 September 2011

\begin{abstract}
Context. Our knowledge of the presence and the role of magnetic fields in intermediate-mass pre-main-sequence stars remains very poor.

Aims. We present the magnetic properties of four Herbig Ae/Be stars that have not been previously studied in detail.

Methods. Our results for the three Herbig Ae/Be stars HD 97048, HD 150193, and HD 176386 are based on multi-epoch lowresolution spectra obtained in spectropolarimetric mode with FORS 2 mounted on the VLT. New high-resolution polarimetric spectra were obtained for MWC 480 with the SOFIN spectrograph installed at the Nordic Optical Telescope. We discuss 41 FORS 2 lowresolution observations of the Herbig Ae/Be stars HD 97048, HD 150193, and HD 176386 and determine their rotational periods. Using stellar fundamental parameters and the longitudinal magnetic field phase curves, we place constraints on the magnetic field geometry. Three high-resolution circularly polarised SOFIN spectra obtained for MWC 480 were measured using the moment technique where wavelength shifts between right- and left-hand side circularly polarised spectra are interpreted in terms of a longitudinal magnetic field $\left\langle B_{z}\right\rangle$.

Results. Our search for periodicities resulted in $P=0.693 \mathrm{~d}$ for HD 97048, $P=1.317 \mathrm{~d}$ for HD 150193, and $P=0.899 \mathrm{~d}$ for HD 176386. The magnetic field geometry can likely be described by a centred dipole with a polar magnetic field strength $B_{\mathrm{d}}$ of several hundred Gauss. The longitudinal magnetic-field measurements of MWC 480 reveal the presence of a strong kG field, which was undetected in our previous low-resolution polarimetric observations with FORS 1. A weak magnetic field was detected in the circumstellar components of the $\mathrm{Ca}$ II $\mathrm{H} \& \mathrm{~K}$ lines and the $\mathrm{Na}$ I D lines, indicating a complex interaction between the stellar magnetic field and the circumstellar environment.
\end{abstract}

Key words. stars: pre-main sequence - stars: atmospheres - stars: magnetic field - stars: variables: general - stars: general

\section{Introduction}

Spectropolarimetric observations of several Herbig Ae/Be stars have indicated that magnetic fields are important ingredients of the intermediate-mass star formation process. Models of magnetically driven accretion and outflows successfully reproduce many observational properties of low-mass pre-main sequence stars (the classical T Tauri stars), but the picture is less clear for higher mass stars. It is important to understand how the magnetic fields in Herbig Ae/Be stars are generated and how these fields interact with the circumstellar environment, displaying a combination of disk, wind, accretion, and jets. To date, these fields have not been studied for a representative number of Herbig Ae/Be stars. Magnetically controlled behaviour has been found in both the spectroscopic and photometric variability of

\footnotetext{
* Based on observations obtained at the European Southern Observatory (ESO programme 085.C-0137(A)) and SOFIN observations at the 2.56-m Nordic Optical Telescope on La Palma.
}

the strongly magnetic Herbig Ae star HD 101412 (Hubrig et al. 2010, 2011). The small amount of UVES spectra acquired for this star have been analysed uncovering variations in equivalent widths, radial velocities, line widths, line asymmetries, and mean magnetic field modulus over the rotation period of $42.1 \mathrm{~d}$. Magnetic field configurations are obviously of utmost importance to understanding the magnetospheres of Herbig $\mathrm{Ae} / \mathrm{Be}$ stars. Since the accreting material has to be lifted from the disk midplane to higher stellar latitudes near the stellar photosphere, some coupling between the accreting plasma and a stellar magnetic field is required. Progress in understanding the diskmagnetosphere interaction can, however, only come from studying a sufficient number of targets in detail to look for patterns encompassing this type of pre-main sequence stars.

In this work, we present series of mean longitudinal magnetic-field measurements for the Herbig $\mathrm{Ae} / \mathrm{Be}$ stars HD 97048, HD 150193, and HD 176386 obtained at low resolution with the multi-mode instrument FORS 2 at the VLT, and 
Table 1. Target stars with multi-epoch spectropolarimetric observations.

\begin{tabular}{lccc}
\hline \hline $\begin{array}{l}\text { Object } \\
\text { name }\end{array}$ & $\begin{array}{c}\text { Other } \\
\text { identifier }\end{array}$ & $V$ & $\begin{array}{c}\text { Spectral } \\
\text { type }\end{array}$ \\
\hline MWC 480 & HD 31648 & 7.7 & A3Ve \\
HD 97048 & CU Cha & 8.5 & A0pshe \\
HD 150193 & V2307 Oph & 8.9 & A1Ve \\
HD 176386 & CD-37 13023 & 7.3 & B9V \\
\hline
\end{tabular}

Notes. Spectral types and visual magnitudes are taken from SIMBAD.

describe their magnetic field geometries. Magnetic fields of the order of 120-250G were detected in these stars for the first time during our visitor run with FORS 1 in May 2008 (Hubrig et al. 2009). The follow-up program developed to search for magnetic field variations over the rotation cycle was allocated for observations with FORS 2 in 2010. The previous spectropolarimetric observations of the star MWC 480 (=HD 31648) with FORS 1 revealed the presence of a weak longitudinal magnetic field $\left\langle B_{z}\right\rangle=87 \pm 22 \mathrm{G}$ and distinct circular polarisation signatures in spectral lines originating in the circumstellar (CS) environment (Hubrig et al. 2006, 2007). We report here on three new high-resolution polarimetric spectra obtained with the SOFIN spectrograph installed at the Nordic Optical Telescope, which confirm the presence of a magnetic field in this star in both photospheric and CS lines.

\section{Magnetic field measurements and period determination}

The targets for which we present new spectropolarimetric measurements are listed in Table 1. Multi-epoch polarimetric spectra of the Herbig Ae/Be stars HD 97048, HD 150193, and HD 176386 were obtained with FORS $2^{1}$ on Antu (UT1) from 2010 March 23 to 2010 June 7 in service mode. Using a slit width of 0.4 , the spectral resolving power of FORS 2 achieved with the GRISM 600B is about 2000. A detailed description of the assessment of the longitudinal magnetic-field measurements using FORS 2 was presented in our previous papers (e.g., Hubrig et al. 2004a,b, and references therein). The mean longitudinal magnetic field, $\left\langle B_{z}\right\rangle$, was derived using

$\frac{V}{I}=-\frac{g_{\mathrm{eff}} e \lambda^{2}}{4 \pi m_{\mathrm{e}} c^{2}} \frac{1}{I} \frac{\mathrm{d} I}{\mathrm{~d} \lambda}\left\langle B_{z}\right\rangle$,

where $V$ is the Stokes parameter that measures the circular polarisation, $I$ is the intensity in the unpolarised spectrum, $g_{\text {eff }}$ is the effective Landé factor, $e$ is the electron charge, $\lambda$ is the wavelength, $m_{\mathrm{e}}$ the electron mass, $c$ the speed of light, $\mathrm{d} I / \mathrm{d} \lambda$ is the derivative of Stokes $I$, and $\left\langle B_{z}\right\rangle$ is the mean longitudinal magnetic field.

Three spectropolarimetric observations of MWC 480 with $S / N \geq 200$ were obtained on 2009 December 30 and 2010 December 14, and 2010 December 25 with the low-resolution camera $(R=\lambda / \Delta \lambda \approx 30000)$ of the echelle spectrograph SOFIN (Tuominen et al. 1999) mounted at the Cassegrain focus of the Nordic Optical Telescope (NOT). We used a 2K Loral CCD detector to register 40 echelle orders partially covering the range from 3500 to $10000 \AA$ with a length of the spectral orders of about $140 \AA$ at $5500 \AA$. The polarimeter is located in front of

\footnotetext{
1 The spectropolarimetric capabilities of FORS 1 were moved to FORS 2 in 2009.
}

Table 2. Magnetic field measurements of Herbig Ae/Be stars with FORS $1 / 2$.

\begin{tabular}{|c|c|c|c|}
\hline MJD & Phase & $\overline{\left\langle\left\langle B_{z}\right\rangle_{\text {all }}[\mathrm{G}]\right.}$ & $\overline{\left\langle B_{z}\right\rangle_{\text {hyd }}[\mathrm{G}]}$ \\
\hline \multicolumn{4}{|c|}{ HD 97048} \\
\hline 54609.137 & 0.083 & $164 \pm 42$ & $188 \pm 47$ \\
\hline 55279.131 & 0.349 & $-92 \pm 41$ & $-135 \pm 58$ \\
\hline 55311.109 & 0.467 & $-163 \pm 53$ & $-195 \pm 59$ \\
\hline 55312.035 & 0.802 & $-6 \pm 74$ & $12 \pm 91$ \\
\hline 55320.094 & 0.426 & $-44 \pm 54$ & $-62 \pm 62$ \\
\hline 55322.119 & 0.346 & $-123 \pm 42$ & $-62 \pm 48$ \\
\hline 55324.105 & 0.210 & $4 \pm 59$ & $9 \pm 63$ \\
\hline 55326.987 & 0.367 & $-124 \pm 34$ & $-156 \pm 44$ \\
\hline 55334.039 & 0.537 & $-154 \pm 53$ & $-193 \pm 61$ \\
\hline 55335.119 & 0.094 & $127 \pm 120$ & $135 \pm 49$ \\
\hline 55345.987 & 0.768 & $54 \pm 59$ & $26 \pm 63$ \\
\hline 55347.180 & 0.488 & $-186 \pm 61$ & $-231 \pm 64$ \\
\hline 55348.070 & 0.772 & $-36 \pm 38$ & $-12 \pm 64$ \\
\hline 55349.102 & 0.260 & $-7 \pm 74$ & $65 \pm 78$ \\
\hline \multicolumn{4}{|c|}{ HD 150193} \\
\hline 54609.092 & 0.118 & $-144 \pm 32$ & $-252 \pm 48$ \\
\hline 55321.364 & 0.523 & $-124 \pm 60$ & $-184 \pm 62$ \\
\hline 55312.319 & 0.648 & $-97 \pm 84$ & $-239 \pm 102$ \\
\hline 55320.179 & 0.622 & $-148 \pm 69$ & $-147 \pm 80$ \\
\hline 55322.295 & 0.230 & $-109 \pm 48$ & $-111 \pm 64$ \\
\hline 55323.311 & 0.003 & $-39 \pm 34$ & $-43 \pm 46$ \\
\hline 55324.285 & 0.744 & $-107 \pm 43$ & $-124 \pm 64$ \\
\hline 55325.307 & 0.520 & $-189 \pm 45$ & $-228 \pm 59$ \\
\hline 55326.341 & 0.306 & $-142 \pm 53$ & $-176 \pm 70$ \\
\hline 55327.340 & 0.066 & $-19 \pm 36$ & $-15 \pm 48$ \\
\hline 55328.356 & 0.838 & $-62 \pm 74$ & $-46 \pm 99$ \\
\hline 55334.314 & 0.366 & $-143 \pm 32$ & $-132 \pm 74$ \\
\hline 55341.279 & 0.661 & $-217 \pm 58$ & $-235 \pm 74$ \\
\hline 55348.125 & 0.864 & $-120 \pm 96$ & $-306 \pm 130$ \\
\hline 55354.296 & 0.555 & $-201 \pm 55$ & $-229 \pm 82$ \\
\hline \multicolumn{4}{|c|}{ HD 176386} \\
\hline 54610.272 & 0.174 & $-119 \pm 33$ & $-121 \pm 35$ \\
\hline 55312.353 & 0.145 & $-10 \pm 69$ & $-42 \pm 88$ \\
\hline 55322.313 & 0.224 & $-41 \pm 65$ & $-44 \pm 89$ \\
\hline 55323.247 & 0.263 & $-62 \pm 62$ & $-126 \pm 64$ \\
\hline 55324.308 & 0.444 & $-190 \pm 62$ & $-179 \pm 65$ \\
\hline 55325.331 & 0.582 & $-201 \pm 63$ & $-225 \pm 71$ \\
\hline 55326.365 & 0.732 & $-115 \pm 54$ & $-119 \pm 68$ \\
\hline 55327.244 & 0.709 & $-6 \pm 64$ & $-49 \pm 79$ \\
\hline 55328.375 & 0.967 & $99 \pm 59$ & $111 \pm 79$ \\
\hline 55334.339 & 0.602 & $-65 \pm 49$ & $-50 \pm 63$ \\
\hline 55335.264 & 0.631 & $-102 \pm 43$ & $-144 \pm 54$ \\
\hline 55341.317 & 0.364 & $-101 \pm 65$ & $-104 \pm 71$ \\
\hline 55349.128 & 0.052 & $115 \pm 50$ & $121 \pm 68$ \\
\hline 55354.226 & 0.724 & $-130 \pm 41$ & $-162 \pm 62$ \\
\hline 55355.134 & 0.733 & $-25 \pm 38$ & $-30 \pm 61$ \\
\hline
\end{tabular}

the entrance slit of the spectrograph and consists of a fixed calcite beam splitter aligned along the slit and a rotating superachromatic quarter-wave plate. Two spectra polarised in opposite senses are recorded simultaneously for each echelle order providing sufficient separation by the cross-dispersion prism. Usually two sub-exposures with the quarter-wave plate angles separated by $90^{\circ}$ are necessary to derive circularly polarised spectra. The spectra are reduced with the $4 \mathrm{~A}$ software package (Ilyin 2000). Bias subtraction, master flat-field correction, scattered light subtraction, and weighted extraction of spectral orders comprise the standard steps of the image processing. A ThAr spectral lamp is used for wavelength calibration, taken before and after each target exposure to minimise temporal variations in the spectrograph. Wavelength shifts between right- and left-hand side circularly polarised spectra were interpreted in 
terms of a longitudinal magnetic field $\left\langle B_{z}\right\rangle$, using the moment technique described by Mathys (1994).

The frequency analysis was performed on the longitudinal magnetic field measurements using a non-linear least squares fit to the multiple harmonics utilizing the Levenberg-Marquardt method (Press et al. 1992) with an optional possibility of prewhitening the trial harmonics. To detect the most probable period, we calculated the frequency spectrum and for each trial frequency we performed a statistical F-test of the null hypothesis for the absence of periodicity (Seber 1977). The resulting F-statistics can be thought of as the total sum including covariances of the ratio of harmonic amplitudes to their standard deviations, i.e. a signal-to-noise ratio. The derived ephemeris for the detected periods are

$$
\begin{aligned}
& \text { HD } 97048:\left\langle B_{z}\right\rangle^{\text {pos ext }}= \\
& \text { MJD55318.41923 } \pm 0.01372+0.69334 \pm 0.00039 E \\
& \text { HD } 150193:\left\langle B_{z}\right\rangle^{\text {pos ext }}= \\
& \text { MJD55318.05538 } \pm 0.03072+1.31697 \pm 0.00013 E \\
& \text { HD } 176386:\left\langle B_{z}\right\rangle^{\text {pos ext }}= \\
& \text { MJD 55325.70578 } \pm 0.02185+0.89920 \pm 0.00008 E \text {. }
\end{aligned}
$$

The logbook of the new FORS 2 and the old FORS 1 spectropolarimetric observations is presented in Table 2. In the first column, we indicate the MJD value at mid exposure. The phases of the measurements of the magnetic field are listed in Col. 2. In Cols. 3 and 4, we present the longitudinal magnetic field $\left\langle B_{z}\right\rangle_{\text {all }}$ measured using the whole spectrum and the longitudinal magnetic field $\left\langle B_{z}\right\rangle_{\text {hyd }}$ using only the hydrogen lines. All quoted errors are $1 \sigma$ uncertainties. The FORS 1 measurements published by Hubrig et al. (2009) are presented in the first line, respectively. Phase diagrams of the data folded with the determined periods are presented in Fig. 1. The quality of our fits is described by a reduced $\chi^{2}$-value that is 0.71 for HD 98048, 0.32 for HD 150193, and 0.92 for HD 176386.

All three Herbig Ae/Be stars exhibit a single-wave variation in the longitudinal magnetic field during the stellar rotation cycle. These observations are usually considered as evidence of a dominant dipolar contribution to the magnetic field topology. Assuming that the studied Herbig Ae/Be stars are oblique dipole rotators, the magnetic dipole axis tilt $\beta$ is constrained by $\left\langle B_{z}\right\rangle^{\max } /\left\langle B_{z}\right\rangle^{\min }=\cos (i+\beta) / \cos (i-\beta)$, where the inclination angle $i$ is derived from resolved observations of their disks. In Table 3, we show for each star in rows 1 and 2 the mean value $\overline{\left\langle B_{z}\right\rangle}$ and the amplitude of the field variation $A_{\left\langle B_{z}\right\rangle}$. For the star HD 97048, Lagage et al. (2006) used VISIR observations of the mid-infrared emission of polycyclic hydrocarbons at the surface of the disk to determine $i=42.8 \pm 2.5^{\circ}$. Using this value, we obtain a magnetic obliquity of $\beta=84.8 \pm 5.0^{\circ}$ and the dipole strength $B_{\mathrm{d}}=720 \pm 120 \mathrm{G}$. The disk inclination of HD 150193, $i=38 \pm 9^{\circ}$, was determined by Fukagawa et al. (2003) using Subaru near-infrared imaging. With this value, we find a magnetic obliquity $\beta=42 \pm 10^{\circ}$ and the dipole strength $B_{\mathrm{d}}=590 \pm 40 \mathrm{G}$.

For HD 176386, the inclination angle $i$ is not known because both IR (Siebenmorgen et al. 2000) and far-UV spectroscopy (Martin-Zaïdi et al. 2008) suggest that the circumstellar matter has the form of an envelope rather than the form of a disk. To constrain the magnetic field geometry of this star, we determined that $v \sin i=165 \pm 8 \mathrm{~km} \mathrm{~s}^{-1}$ from our NTT/EMMI spectrum obtained for this star in 1995 July 8. In Fig. 2, we present the line profile of $\mathrm{Mg}$ II 4481 overplotted by the corresponding synthetic spectrum assuming $T_{\text {eff }}=9900 \mathrm{~K}$ and $\log g=3.7$,
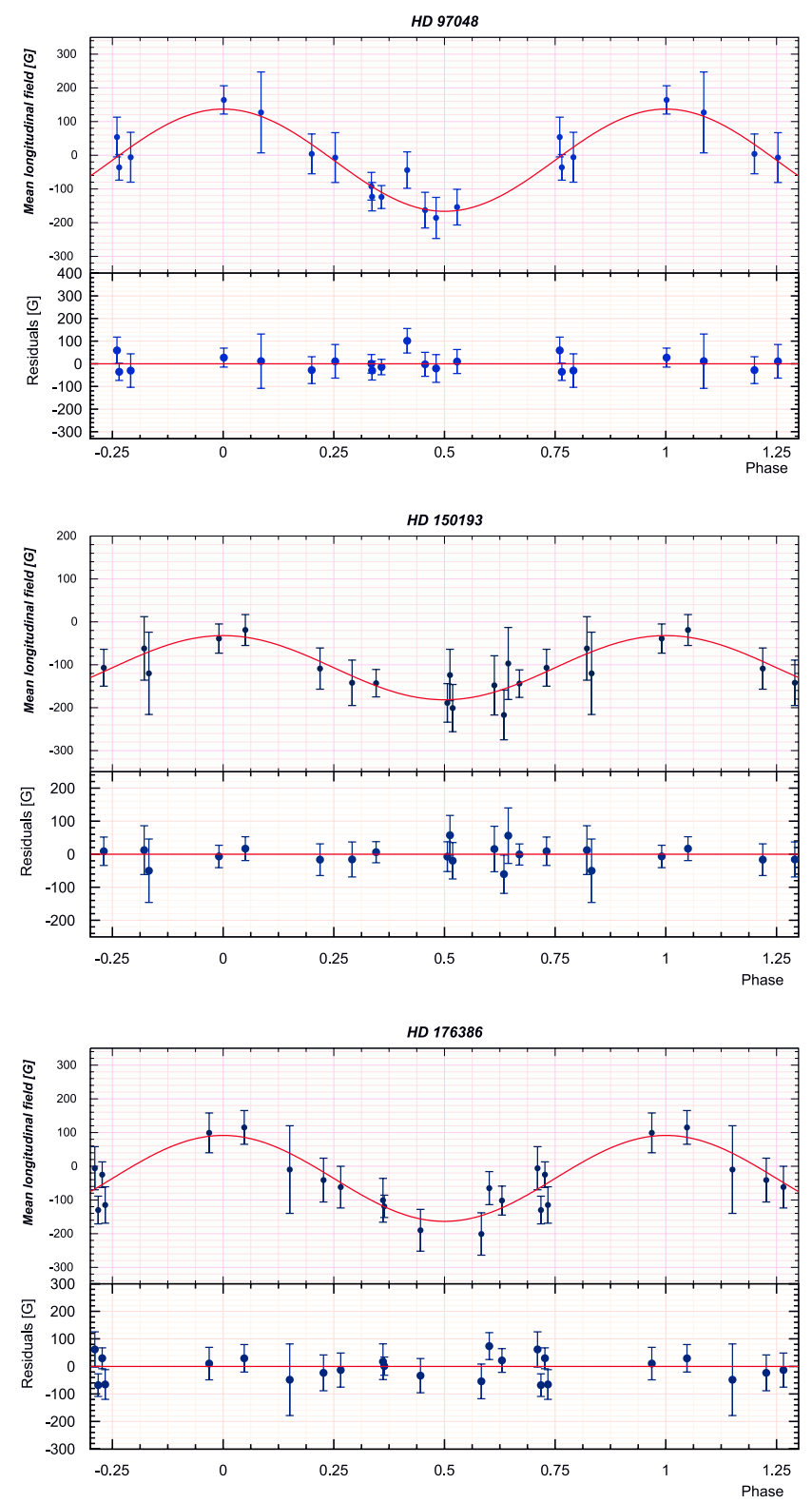

Fig. 1. Phase diagrams with the best sinusoidal fit for the longitudinal magnetic field measurements of HD 97048 (top), HD 150193 (middle), and HD 176386 (bottom). The residuals (observed - calculated) are shown in the lower panel. The deviations are mostly of the same order as the error bars, and no systematic trends are obvious, which justifies a single sinusoid as a fit function.

and solar abundances. We note that the literature values for $T_{\text {eff }}$ widely differ from each other, from $T_{\text {eff }}=10715 \mathrm{~K}$ as given by van den Ancker et al. (1997) to $T_{\text {eff }}=9527 \mathrm{~K}$ derived by Meyer \& Wilking (2006). The equatorial rotation velocity can be calculated using the relation $v_{\mathrm{eq}}=50.6 R / P$, where $R$ is the stellar radius in solar units and $P$ is the rotation period in days. Taking into account the uncertainties in atmospheric parameter determinations for HD 176386, we adopt the radius $R=3 \pm 0.5 R_{\odot}$. With this radius and the period $P=0.899 \mathrm{~d}$, we obtain $v_{\text {eq }}=169 \pm 28 \mathrm{~km} \mathrm{~s}^{-1}$, which leads to the poorly constrained $i=78 \pm 46^{\circ}$, magnetic obliquity $\beta=37 \pm 106^{\circ}$, and the dipole strength $B_{\mathrm{d}}=700 \pm 650 \mathrm{G}$. The parameters of the magnetic field dipole models for all three stars are listed in the last two rows of Table 3. 
Table 3. Magnetic field models constrained by the FORS 1/2 spectropolarimetric observations.

\begin{tabular}{cc|ccc}
\hline \hline \multicolumn{2}{c|}{ Object } & HD 97048 & HD 150193 & HD 176386 \\
\hline$\overline{\left\langle B_{z}\right\rangle}$ & {$[\mathrm{G}]$} & $-14.8 \pm 14.3$ & $-106.9 \pm 7.4$ & $-36.2 \pm 15.9$ \\
$A_{\left\langle B_{z}\right\rangle}$ & {$[\mathrm{G}]$} & $151.6 \pm 19.6$ & $74.8 \pm 9.8$ & $127.5 \pm 24.7$ \\
$v \sin i$ & {$\left[\mathrm{~km} \mathrm{~s}^{-1}\right]$} & & & $165 \pm 8$ \\
$R$ & {$\left[R_{\odot}\right]$} & & & $3.0 \pm 0.5$ \\
$v_{\mathrm{eq}}$ & {$\left[\mathrm{km} \mathrm{s}^{-1}\right]$} & & & $169 \pm 28$ \\
$i$ & {$\left[{ }^{\circ}\right]$} & $42.8 \pm 2.5$ & $38 \pm 9$ & $78 \pm 46$ \\
$\beta$ & {$\left[{ }^{\circ}\right]$} & $84.8 \pm 5.0$ & $41.8 \pm 10.1$ & $37 \pm 106$ \\
$B_{\mathrm{d}}$ & {$[\mathrm{G}]$} & $720 \pm 120$ & $590 \pm 40$ & $700 \pm 650$ \\
\hline
\end{tabular}

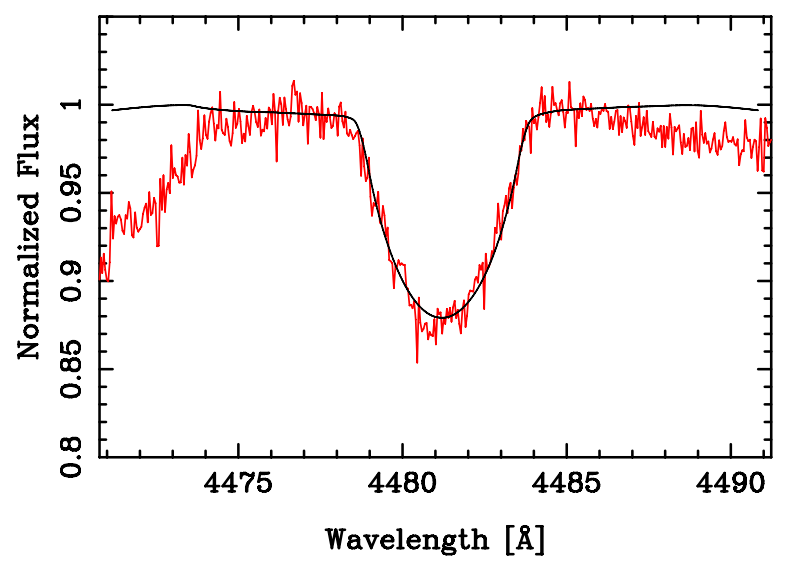

Fig. 2. Determination of the $v \sin i$ value for HD 176386. The line profile of $\mathrm{Mg}_{\text {II }} 4481$ in the NTT/EMMI spectrum is fitted by the corresponding synthetic spectrum assuming $T_{\text {eff }}=9900 \mathrm{~K}$ and $\log g=3.7$.

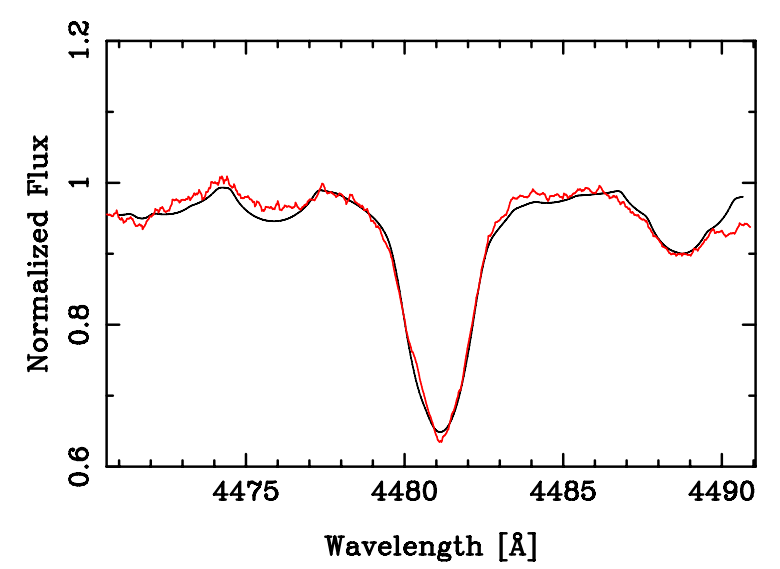

Fig. 3. Determination of the $v \sin i$ value for MWC 480. The line profile of $\mathrm{Mg}$ II 4481 in the SOFIN spectrum is fitted by the corresponding synthetic spectrum assuming $T_{\text {eff }}=8500 \mathrm{~K}$ and $\log g=3.5$.

Table 4. Magnetic field measurements of MWC 480 with SOFIN.

\begin{tabular}{lc}
\hline \hline MJD & $\left\langle B_{z}\right\rangle[\mathrm{G}]$ \\
\hline 53296.350 & $87 \pm 22$ \\
55195.086 & $-952 \pm 177$ \\
55544.090 & $458 \pm 149$ \\
55555.982 & $189 \pm 204$ \\
\hline
\end{tabular}

The logbook of our new SOFIN spectropolarimetric observations of MWC 480 is presented in Table 4. In the first column, we list the MJD values for the middle of the spectropolarimetric observations and in Col. 2 we present the mean longitudinal magnetic field $\left\langle B_{z}\right\rangle$. In the first line, we list the earlier FORS 1

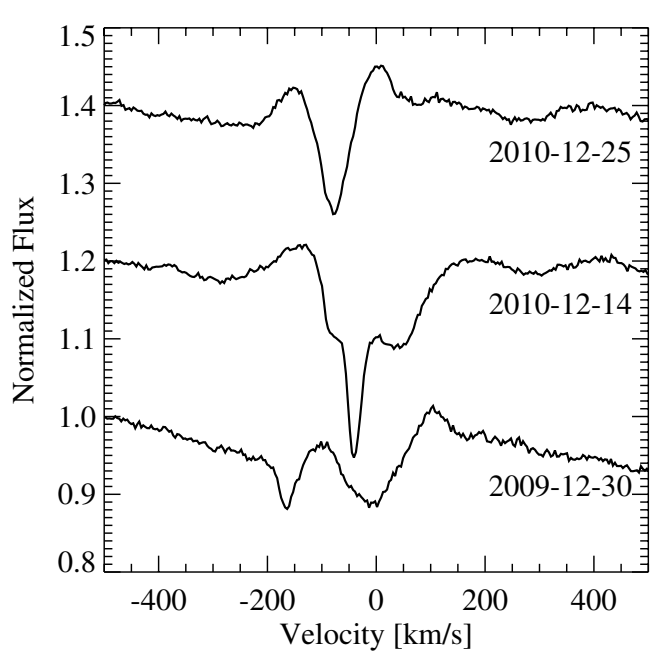

Fig. 4. The behaviour of the $\mathrm{Fe}$ in line at $\lambda 5018.4$ at the three observational epochs 2009 December 30 (bottom), 2010 December 14 (middle), and 2010 December 25 (top).

measurement published by Hubrig et al. (2006). All quoted errors are $1 \sigma$ uncertainties. Since only four magnetic field measurements are available, no search for a periodicity can be undertaken. On the other hand, the rotation period can be estimated using the known inclination angle $i=36 \pm 1^{\circ}$ (Piétu et al. 2006), the radius $R=1.67 R_{\odot}$ (Blondel \& Tijn A Djie 2006), and $v \sin i=90 \pm 2 \mathrm{~km} \mathrm{~s}^{-1}$ estimated on our SOFIN spectra. In Fig. 3, we present the line profile of $\mathrm{Mg}$ II 4481 overplotted by the synthetic spectrum assuming $T_{\text {eff }}=8500 \mathrm{~K}$ and $\log g=3.5$. With this value for $v \sin i$, we obtain $v_{\mathrm{eq}}=153 \pm 8 \mathrm{~km} \mathrm{~s}^{-1}$. By substituting these values into the relation $P=50.6 R / v_{\text {eq }}$, it follows that $P=0.531 \pm 0.029 \mathrm{~d}$. This period is probably very close to the true rotation period, since Grady et al. (2010) report that their FUSE observations integrated over the individual FUSE orbits indicate a marginal detection of variability on the timescale of $0.5 \mathrm{~d}$.

\section{Magnetic field and CS environment of MWC 480}

In our earlier studies based on the low-resolution polarimetric FORS 1 spectra, we reported the detection of distinct Zeeman features in several Herbig Ae/Be stars at the positions of the Ca II H\&K lines (Hubrig et al. 2004c, 2006, 2007, 2009). The Ca II H\&K lines frequently display variable multi-component complex structures in both the Stokes $V$ and Stokes $I$ spectra and it has been suggested that they originate at the base of the stellar wind, as well as in the gaseous accretion flow. The star 

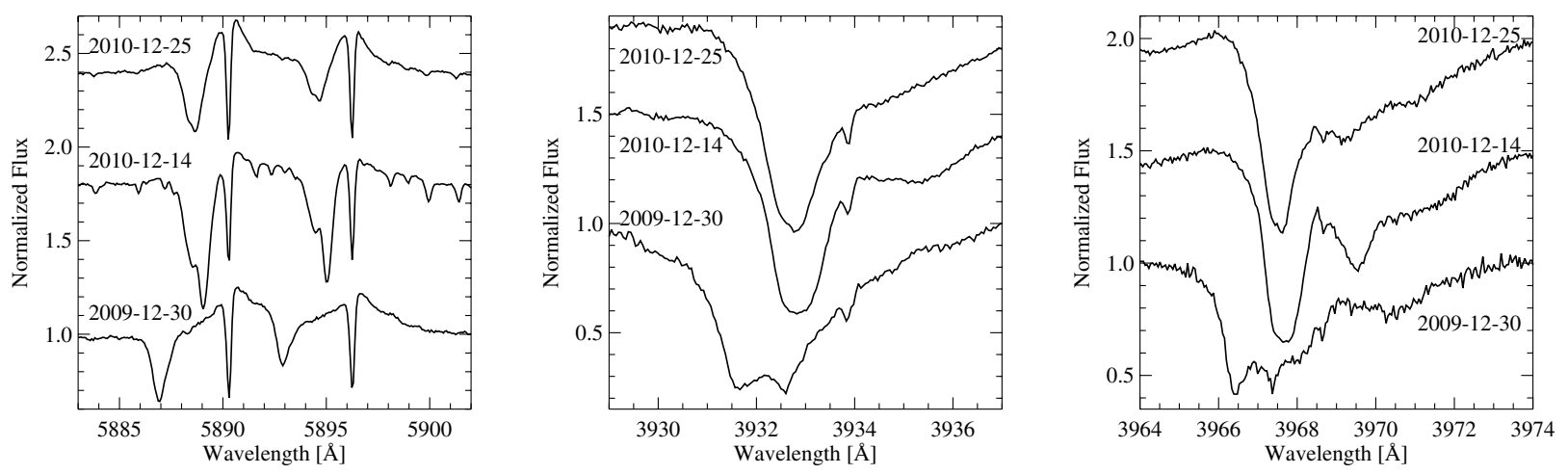

Fig. 5. The variability and complex structure of $\mathrm{Na}$ I D (left) and Ca II K\&H (middle and right) in the spectra of MWC 480 at the same observational epochs as in Fig. 4.

MWC 480 exhibits a significant spectral variability, which implies that there is a strong interaction between the star and its CS environment. The temporal behaviour of the complex variable structure of $\mathrm{H} \alpha, \mathrm{He}$, and the $\mathrm{Na}$ I D line was monitored in several previous studies (e.g., Beskrovnaya \& Pogodin 2004; Kozlova et al. 2007), which showed that the stellar wind is non-homogeneous and consists of several components, differing from each other in terms of their velocities. Furthermore, structural variations in the stellar wind have been found to correlate with changes in the accretion process in the envelope.

In our high-resolution SOFIN spectra, the contamination of the spectra by the CS material is clearly visible. In Fig. 4, we show the presence of a sharp blue component in the Mult. 42 Fe II line at $\lambda 5018.4$ during the first and the second observational epochs in 2009 and 2010. During the last observation, the wings of the $\mathrm{Fe}$ II line appear in emission. In addition, two other lines, $\lambda \lambda 5169.0$ and 4923.9 of the same multiplet, exhibit a similar behaviour. The shift of this sharp blue component, reaches $\sim 153 \mathrm{~km} \mathrm{~s}^{-1}$ during the first SOFIN observation in 2009, and is of the same order as the shifts measured for the blue components of the $\mathrm{Na}$ I D, the $\mathrm{Ca}$ II $\mathrm{H} \& \mathrm{~K}$ lines, and the cores of the hydrogen lines.

In agreement with previous studies, the $\mathrm{Na}$ I $\mathrm{D}$ lines are highly variable, but the $\mathrm{Ca}$ II $\mathrm{H} \& \mathrm{~K}$ lines also show a complex structure consisting of several components and pronounced variability. The behaviour of the $\mathrm{Na}$ I and $\mathrm{Ca}$ II doublets at three different observation epochs is presented in Fig. 5. Owing to strong blending, it is impossible to measure the shift between the line profiles in right- and left-hand side circularly polarised spectra for all line components. The compilation of measurements of the component positions in the $\mathrm{Ca}$ II and $\mathrm{Na}$ I doublet lines and our attempts to determine the longitudinal magnetic field are presented in Table A.1.

Weak magnetic fields of the order of several tens of Gauss were detected in the CS components of the $\mathrm{Na}$ I and $\mathrm{Ca}$ II doublet lines and can probably be considered as CS magnetic fields. If we assume a dipole configuration for the magnetic field geometry, then we expect to measure a magnetic field strength of $-76 \mathrm{G}$ detected in the $\mathrm{CS}$ component of the $\mathrm{Ca}$ II $\mathrm{K}$ line at a distance of $\sim 2.3$ stellar radii. This consideration is fully in line with the generally assumed scenario of magnetospheric accretion in Herbig Ae and T Tauri stars. The stellar magnetic field truncates the accretion disk at a few stellar radii and gas accretes along magnetic channels from the protoplanetary disk to the star. The disk is truncated at a radius $R_{\mathrm{T}}$, which depends upon the strength

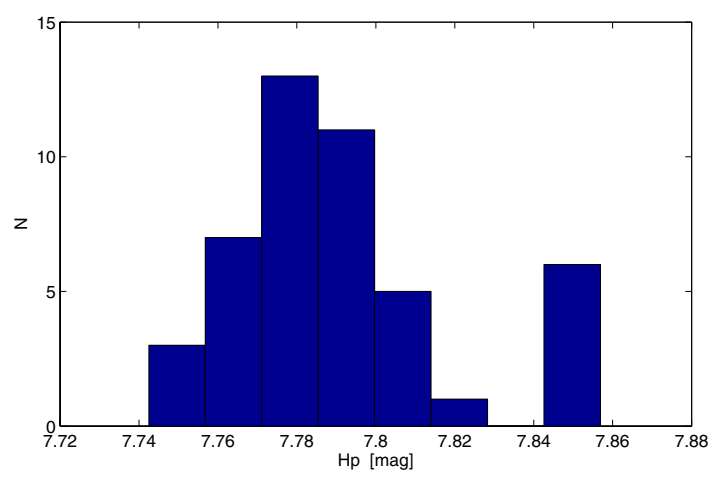

Fig. 6. Available HiPParcos measurements for MWC 480 with outliers observed during HJD 8674.75-8674.94.

of the surface magnetic field, the mass accretion radius, the stellar mass, and the stellar radius (e.g., Bouvier et al. 2007). For a dipole configuration of the magnetic field geometry, a magnetic field strength of $-120 \mathrm{G}$ detected in the CS component of the $\mathrm{Na}$ I $\mathrm{D}$ is expected to be measured at a distance of $\sim 2.0$ stellar radii.

\section{Discussion}

Using FORS 2 low-resolution multi-epoch polarimetric spectra, we have determined rotation/magnetic periods for the three Herbig Ae/Be stars HD 97048, HD 150193, and HD 176386. In the framework of the rigid rotator model usually assumed for magnetic stars on the upper main-sequence, the period of the magnetic field variation corresponds to the stellar rotation period. Our study indicates that dipole models provide a satisfactory fit to the acquired magnetic data.

On the other hand, our search for rotation-modulated photometric variability using the available photometric databases has been unsuccessful: no clear variation was found for the three stars in either the ASAS database (Pojmański 2002) or HipParcos photometry. The star MWC 480 was not observed by ASAS, and only HIPPARcos photometry is available for a period search. It displayed moderate light changes of up to 0.098 mag on timescales from tens of hours to several days. The behaviour of MWC 480 resembles the characteristics typical of other photometrically monitored Herbig Ae/Be stars, for which light variations are likely of stochastic nature and caused by 

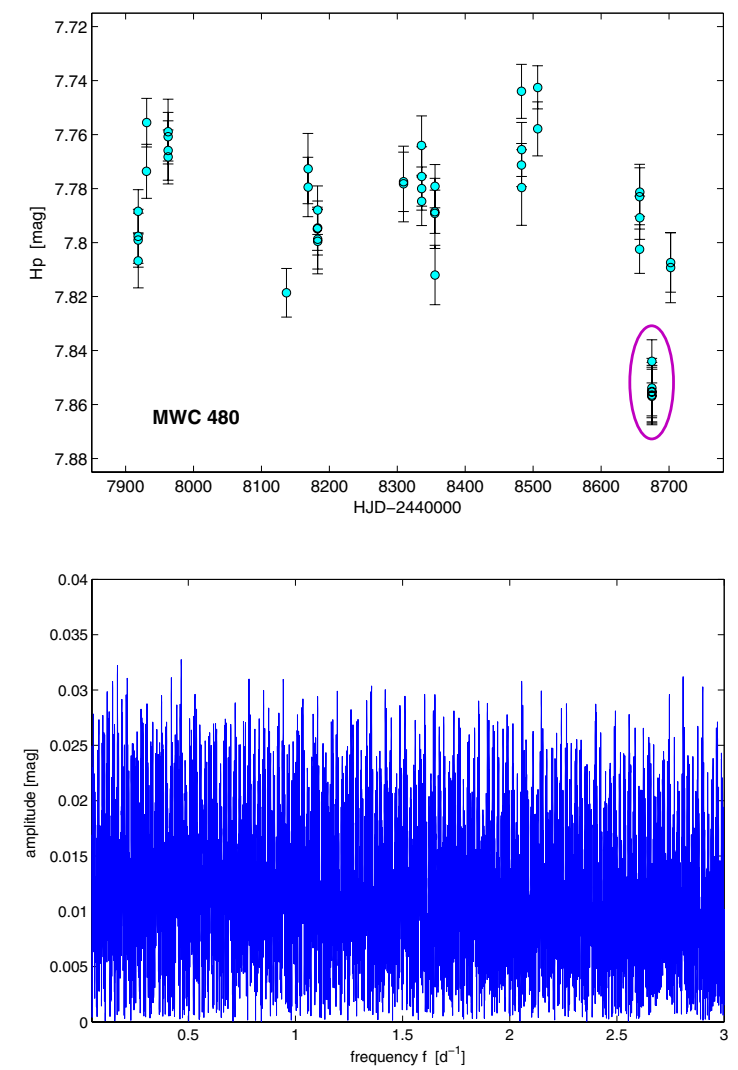

Fig. 7. Hipparcos observations of MWC 480 and the corresponding frequency periodogram. The six outliers are indicated by an ellipse.

the pre-main-sequence disk accretion phenomena (e.g. Rucinski et al. 2010). Using archive Hipparcos data, we have found an indication of a variability with a period of $1.6 \mathrm{~d}$. However, it seems that six outliers appear during the period HJD 8674.758674.94. This group of outliers is located on the right side of the histogram presented in Fig. 6. Excluding these outliers from the analysis, we found no indication for periodicity. In Fig. 7, we present HiPPARcos observations of MWC 480 and the corresponding frequency periodogram.

Kozlova et al. (2007) studied the spectral variability of MWC 480 in the region of $\mathrm{H} \alpha$. They found an equivalent width variability on a timescale of about $1200 \mathrm{~d}$ and suggest that the most plausible reason for the detected cyclic variability is the reconstruction of the inner structure of the CS gas envelope, caused by the presence of a low mass companion or planet around the star. It is not yet clear how and whether this periodicity is related to the appearance of the red-shifted component in the $\mathrm{Ca}$ II $\mathrm{H} \& \mathrm{~K}$ lines during the second and third epochs of the SOFIN observations.

Our experience shows that the photometric periodic variability of Herbig Ae stars is generally difficult to detect. As an example, we show in Fig. 8 Hipparcos photometric data for V380 Ori, for which the rotation period $P=4.31276 \pm 0.00042 \mathrm{~d}$ was determined from magnetic field measurements (Alecian et al. 2009). As we show in this figure, no periodicity can be detected in the photometric data.

Until recently, rotation periods had been known for only three Herbig Ae stars, V380 Ori, HD 101412, and HD 135544B. They had been determined from direct measurements of longitudinal magnetic fields (for V380 Ori and
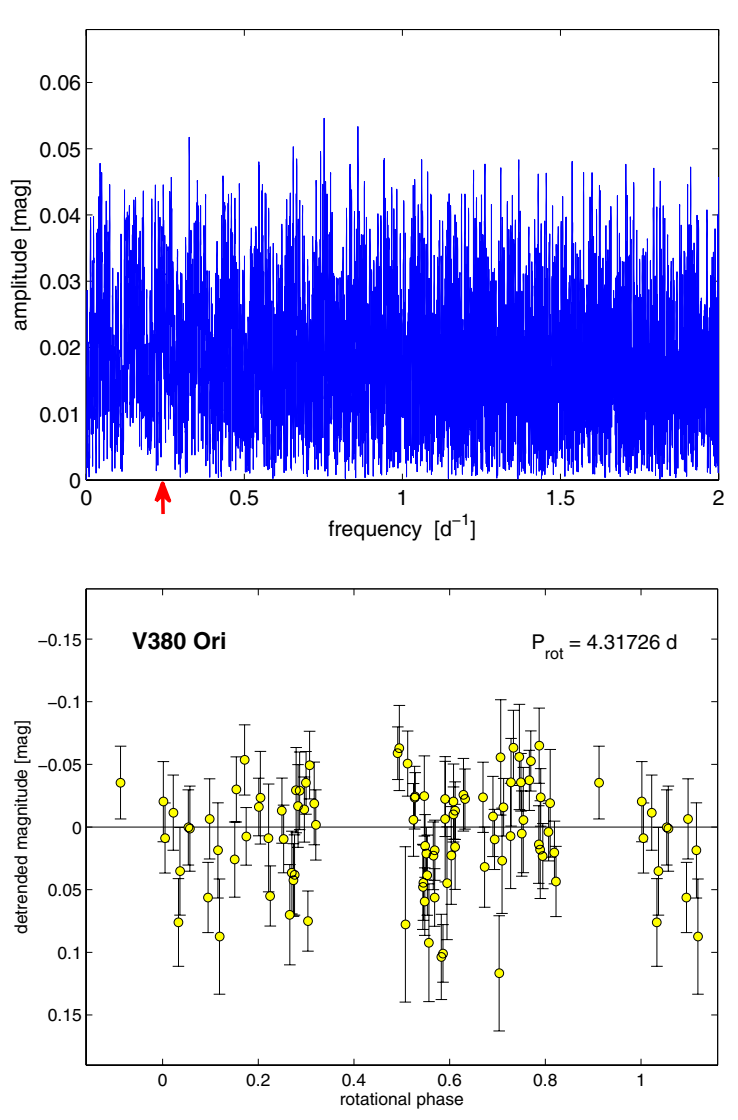

Fig. 8. Frequency periodogram from HIPPARcos observations of V380 Ori. The red arrow in this figure denotes the rotation period determined by Alecian et al. (2009). The lower panel presents the corresponding phase diagram. No periodicity is detected.

HD 101412), photometry (for HD 101412), or radial velocities (for HD 135544B) (Alecian et al. 2009; Hubrig et al. 2011; Müller et al. 2011).

The longitudinal magnetic fields of HD 97048, HD 150193, and HD 176386 are not very strong compared to the $\mathrm{kG}$ fields detected in V380 Ori, HD 101412, and ZCMa (Alecian et al. 2009; Hubrig et al. 2010, 2011; Szeifert et al. 2010). Grady et al. (2010) placed limits on the field strength for MWC 480 assuming that this star is accreting magnetospherically. Their result, $B_{\text {aver }}=3_{-2}^{+5} \mathrm{kG}$, appears comparable with our measurements presented in this work.

The detection of a weak longitudinal magnetic field in the blue-shifted CS components of the $\mathrm{NaI}$ and the $\mathrm{Ca}$ II doublet lines in the polarimetric spectra of MWC 480 implies that for Herbig Ae/Be stars the Doppler-shifted spectropolarimetric contributions from photospheric and circumstellar environmental diagnostic lines should be investigated using high-resolution polarimetric observations over the rotation period. These studies will allow us to apply the technique of Doppler Zeeman tomography to determine the correspondence between the magnetic field structure and the radial density and temperature profiles.

Acknowledgements. This research has made use of the SIMBAD database, operated at CDS, Strasbourg, France. M.A.P. and R.V.Y. acknowledge the support obtained by RFBR grant No 07-02-00535a and Sci.Schole No 6110.2008.2. 
Table A.1. Wavelength shifts for MWC 480 with corresponding velocities and magnetic field measurements of components identified in the Ca II and Na I doublet line profiles.

\begin{tabular}{|c|c|c|c|c|c|c|c|c|c|}
\hline Component & $\begin{array}{c}\Delta \lambda \\
{[\AA]}\end{array}$ & $\begin{array}{c}\text { Epoch } 1 \\
\text { velocity shift } \\
{\left[\mathrm{km} \mathrm{s}^{-1}\right]}\end{array}$ & $\begin{array}{c}\left\langle B_{z}\right\rangle \\
{[\mathrm{G}]} \\
\end{array}$ & $\begin{array}{c}\Delta \lambda \\
{[\AA]}\end{array}$ & $\begin{array}{c}\text { Epoch } 2 \\
\text { velocity shift } \\
{\left[\mathrm{km} \mathrm{s}^{-1}\right]}\end{array}$ & $\begin{array}{c}\left\langle B_{z}\right\rangle \\
{[\mathrm{G}]}\end{array}$ & $\begin{array}{c}\Delta \lambda \\
{[\AA]}\end{array}$ & $\begin{array}{c}\text { Epoch } 3 \\
\text { velocity shift } \\
{\left[\mathrm{km} \mathrm{s}^{-1}\right]}\end{array}$ & $\begin{array}{l}\left\langle B_{z}\right\rangle \\
{[\mathrm{G}]}\end{array}$ \\
\hline \multicolumn{10}{|c|}{ Ca II 3933.7} \\
\hline 1 st & -1.92 & -146.4 & & -0.86 & -65.6 & 17 & & & \\
\hline 2nd & -1.09 & -83.1 & -24 & & & & -0.84 & -64.1 & \\
\hline $3 \mathrm{rd}$ & 0.18 & 13.7 & -30 & 0.18 & 13.7 & & 0.18 & 13.7 & \\
\hline 4th & & & & 1.31: & 99.9: & & & & \\
\hline \multicolumn{10}{|c|}{ Ca II 3968.5} \\
\hline $1 \mathrm{st}$ & -1.92 & -146.4 & -76 & -0.85 & -64.3 & 56 & & & \\
\hline 2nd & -1.09 & -83.1 & -20 & & & & & & \\
\hline $3 \mathrm{rd}$ & & & & 0.17 & 13.0 & & 0.17 & 13.0 & \\
\hline 4th & & & & 1.06: & 80.1: & & 0.67: & 50.7: & \\
\hline \multicolumn{10}{|c|}{$\mathrm{H} \epsilon$} \\
\hline & 0.19 & 14.4 & -706 & & & & & & \\
\hline \multicolumn{10}{|c|}{ Na I 5890.0} \\
\hline $1 \mathrm{st}$ & -3.01 & -152.8 & -116 & -1.43 & -72.8 & & & & \\
\hline 2nd & & & & -0.90 & -45.8 & & -1.31 & -66.7 & \\
\hline $3 \mathrm{rd}$ & 0.28 & 14.3 & -16 & 0.28 & 14.3 & -21 & 0.28 & 14.3 & \\
\hline \multicolumn{10}{|c|}{ Na I 5895.9} \\
\hline $1 \mathrm{st}$ & -2.99 & -152.1 & -90 & -1.44 & -72.8 & & & & \\
\hline 2nd & & & & -0.90 & -45.8 & & -1.3 & -66.2 & \\
\hline $3 \mathrm{rd}$ & 0.30 & 15.2 & -120 & 0.30 & 15.2 & & 0.30 & 15.2 & \\
\hline
\end{tabular}

Notes. Numbers with colons indicate the most uncertain values due to blends.

\section{Appendix A: Magnetic field measurements of components identified in the $\mathrm{Ca} \|$ and $\mathrm{Na}$ । doublet line profiles}

During the first epoch of our SOFIN observations in December 2009 , the Ca doublet lines were found to have three components. Two of them are shifted to the blue by $146.4 \mathrm{~km} \mathrm{~s}^{-1}$ and $83.1 \mathrm{~km} \mathrm{~s}^{-1}$, respectively, and the third one is shifted to the red by $13.7 \mathrm{~km} \mathrm{~s}^{-1}$. This velocity corresponds to the radial velocity of the photospheric spectral lines. During the epochs 2 and 3, an additional strong broad component appears on the red side. The shift measured during the second epoch is almost $100 \mathrm{~km} \mathrm{~s}^{-1}$. Such a strong broad red-shifted component is also discovered in the higher number Balmer lines, providing potential evidence of a lower mass companion. In any case, future studies should focus on multi-epoch spectroscopic observations to monitor the radial velocities and the line profile variations in this star and prove the presence of such a companion. Wavelength shifts for MWC 480 and the corresponding velocities and magnetic field measurements of its components identified in the $\mathrm{Ca}$ II and $\mathrm{Na}$ I doublet line profiles are presented in Table A.1. The blue CS components in the $\mathrm{Na}$ I D lines appear to be clearly separated at the first epoch allowing us to measure a weak longitudinal magnetic field of the order of $\sim-100 \mathrm{G}$. The Na I D lines themselves are in emission. They have overlapping sharp absorption components. During the second and third epochs of observations, the blue CS components indicate a double structure and are located closer to the emission $\mathrm{Na}$ I $\mathrm{D}$ lines.

As for the large shifts in the blue components of the Na I D lines and the Ca II H\&K lines detected in the SOFIN spectrum in 2009, the cores of the hydrogen lines from $\mathrm{H} \beta$ to $\mathrm{H} 8$ in our FORS 1 spectra obtained in 2004 are also shifted to the blue by $\sim 150 \mathrm{~km} \mathrm{~s}^{-1}$. In Fig. A.1, we show the behaviour of the $\mathrm{H} \gamma$ and $\mathrm{H} \delta$ line profiles together with the calculated synthetic spectra for atmosphere model parameters $T_{\text {eff }}=8500 \mathrm{~K}$ and $\log g=$ 3.5. Interestingly, this pattern is the opposite of that seen for the
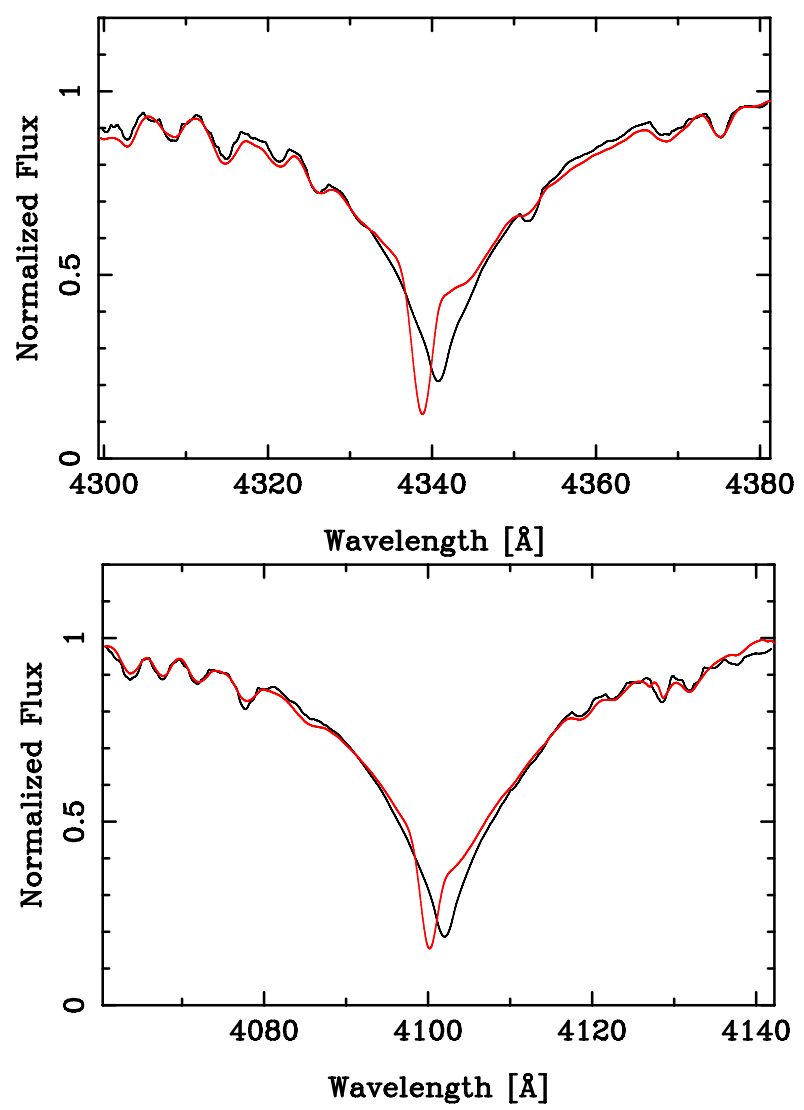

Fig. A.1. The $\mathrm{H} \gamma$ (upper plot) and $\mathrm{H} \delta$ (lower plot) line profiles in the spectrum of MWC 480 observed with FORS 1 in 2004 with an overplotted synthetic spectrum assuming $T_{\text {eff }}=8500 \mathrm{~K}$ and $\log g=3.5$.

Herbig Ae star HD 101412, where the higher order Balmer lines display red shifts that decrease towards the lower order Balmer 
lines. It is possible that the opposite trend for hydrogen line-core velocities in both stars can be explained by different viewing angles, i.e. we view MWC 480 mostly in the wind direction at an angle $i=36 \pm 1^{\circ}$ (Piétu et al. 2006), whereas HD 101412 is viewed in the direction of the accretion flow with $i=80 \pm 7^{\circ}$ (Fedele et al. 2008).

\section{References}

Alecian, E., Wade, G. A., Catala, C., et al. 2009, MNRAS, 400, 354 Beskrovnaya, N. G., \& Pogodin, M. A. 2004, A\&A, 414, 955

Blondel, P. F. C., \& Tjin A Djie, H. R. E. 2006, A\&A, 456, 1045

Bouvier, J., Alencar, S. H. P., Harries, T. J., et al. 2007, Protostars and Planets V, ed. B. Reipurth, D. Jewitt, and K. Keil, 479 (University of Arizona Press: Tucson)

Fedele, D., van den Ancker, M. E., Acke, B., et al. 2008, A\&A, 491, 809 Fukagawa, M., Tamura, M., Itoh, Y., et al. 2003, ApJ, 590, L49

Grady, C. A., Hamaguchi, K., Schneider, G., et al. 2010, ApJ, 719, 1565

Hubrig, S., Kurtz, D. W., Bagnulo, S., et al. 2004a, A\&A, 415, 661

Hubrig, S., Szeifert, T., Schöller, M., et al. 2004b, A\&A, 415, 685

Hubrig, S., Schöller, M., \& Yudin, R. V. 2004c, A\&A, 428, L1

Hubrig, S., Yudin, R. V., Schöller, M., \& Pogodin, M. A. 2006, A\&A, 446, 1089
Hubrig, S., Pogodin, M. A., Yudin, R. V., et al. 2007, A\&A, 463, 1039 Hubrig, S., Stelzer, B., Schöller, M., et al. 2009, A\&A, 502, 283

Hubrig, S., Schöller, M., Savanov, I., et al. 2010, Astron. Nachr., 331, 361

Hubrig, S., Mikulasek, Z., González, J. F., et al. 2011, A\&A, 525, L4

Ilyin, I. 2000, Ph.D. Thesis, University of Oulu, Finland

Kozlova, O. V., Alekseev, I. Yu., \& Shakhovskoi, D. N. 2007, Astrophysics, 50, 467

Lagage, P-O., Doucet, C., Pantin, E., et al. 2006, Science, 314, 621

Martin-Zaïdi, C., Deleuil, M., Le Bourlot, J., et al. 2008, A\&A, 484, 225

Mathys, G. 1994, A\&AS, 108, 547

Müller, A., van den Ancker, M. E., Launhardt, R., et al. 2011, A\&A, 530, A85

Meyer, M. R., \& Wilking, B. A. 2006, PASP, 121, 350

Piétu, V., Dutrey, A., Guilloteau, S., et al. 2006, A\&A, 460, L43

Pojmański, G. 2002, Acta Astron., 52, 397

Press, W. H., Teukolsky, S. A., Vetterling, W. T., \& Flannery, B. P. 1992, Numerical Recipes, 2nd edn. (Cambridge: Cambridge University Press)

Rucinski, S. M., Zwintz, K., Hareter, M., et al. 2010, A\&A, 522, A113

Seber, G. A. F. 1977, Linear Regression Analysis (New York: Wiley)

Siebenmorgen, R., Prusti, T., Natta, A., \& Müller, T. G. 2000, A\&A, 361, 258

Szeifert, T., Hubrig, S., Schöller, M., et al. 2010, A\&A, 509, L7

Tuominen, I., Ilyin, I., \& Petrov, P. 1999, in Astrophysics with the NOT, ed. H. Karttunen, \& V. Piirola, University of Turku, Tuorla Observatory, 47

van den Ancker, M. E., The, P. S., Tjin A Djie, H. R. E., et al. 1997, A\&A, 324, L33 